\title{
The effect of spinal manipulative therapy and home stretching exercises on heart rate variability in patients with persistent or recurrent neck pain: a randomized controlled trial
}

\author{
Anders Galaasen Bakken ${ }^{1 *} \mathbb{0}$, Andreas Eklund ${ }^{1}$, David M. Hallman² and Iben Axén ${ }^{1}$
}

\begin{abstract}
Background: Persistent or recurrent neck pain is, together with other chronic conditions, suggested to be associated with disturbances of the Autonomic Nervous System. Acute effects on the Autonomic Nervous System, commonly measured using Heart Rate Variability, have been observed with manual therapy. This study aimed to investigate the effect on Heart Rate Variability in (1) a combination of home stretching exercises and spinal manipulative therapy versus (2) home stretching exercises alone over 2 weeks in participants with persistent or recurrent neck pain.

Methods: A randomized controlled clinical trial was carried out in five multidisciplinary primary care clinics in Stockholm from January 2019 to April 2020. The study sample consisted of 131 participants with a history of persistent or recurrent neck. All participants performed home stretching exercises daily for 2 weeks and were scheduled for four treatments during this period, with the intervention group receiving spinal manipulative therapy in addition to the home exercises. Heart Rate Variability at rest was measured at baseline, after 1 week, and after 2 weeks, with RMSSD (Root mean square of successive RR interval differences) as the primary outcome. Both groups were blinded to the other group intervention. Thus, they were aware of the purpose of the trial but not the details of the "other" intervention. The researchers collecting data were blinded to treatment allocation, as was the statistician performing data analyses. The clinicians provided treatment for participants in both groups and could not be blinded. A linear mixedeffects model with continuous variables and person-specific random intercept was used to investigate the grouptime interaction using an intention to treat analysis.
\end{abstract}

Results: Sixty-six participants were randomized to the intervention group and sixty-five to the control group. For RMSSD, a B coefficient of 0.4 ( $p$ value: 0.9 ) was found, indicating a non-significant difference in the regression slope for each time point with the control group as reference. No statistically significant differences were found between groups for any of the Heart Rate Variability indices.

\footnotetext{
*Correspondence: Anders.Galaasen.Bakken@ki.se

${ }^{1}$ Department of Environmental Medicine, Division of Intervention

and Implementation Research for Worker Health, Karolinska Institutet,

Nobels väg 13, 17177 Stockholm, Sweden

Full list of author information is available at the end of the article
}

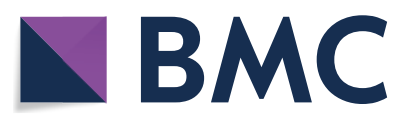

(c) The Author(s) 2021. Open Access This article is licensed under a Creative Commons Attribution 4.0 International License, which permits use, sharing, adaptation, distribution and reproduction in any medium or format, as long as you give appropriate credit to the original author(s) and the source, provide a link to the Creative Commons licence, and indicate if changes were made. The images or other third party material in this article are included in the article's Creative Commons licence, unless indicated otherwise in a credit line to the material. If material is not included in the article's Creative Commons licence and your intended use is not permitted by statutory regulation or exceeds the permitted use, you will need to obtain permission directly from the copyright holder. To view a copy of this licence, visit http://creativecommons.org/licenses/by/4.0/. The Creative Commons Public Domain Dedication waiver (http://creativeco mmons.org/publicdomain/zero/1.0/) applies to the data made available in this article, unless otherwise stated in a credit line to the data. 
Conclusion: Adding four treatments of spinal manipulation therapy to a 2-week program of daily stretching exercises gave no significant change in Heart Rate Variability.

Trial Registration: The trial was registered 03/07/2018 at ClinicalTrials.gov, registration number: NCT03576846. (https:// pubmed.ncbi.nlm.nih.gov/31606042/)

Keywords: Manipulative therapy, Stretching exercises, Heart Rate Variability, HRV, Autonomic nervous system

\section{Introduction}

Chronic pain affects people globally and is estimated to be the reason for $15 \%$ to $20 \%$ of all physician visits [1]. Chronic neck pain (NP) was globally ranked fifth in 2015 measured by years lived with disability [2]. In 2017, the worldwide prevalence of neck pain was found to be 3551 per 100.000 [3]."

Chronic NP is, like all types of chronic pain, a complicated matter. The discussion has reached a point where the term "chronic pain" is now questioned, and other definitions have been suggested $[4,5]$. This study uses persistent or recurrent pain synonymously for chronic pain, because studies have shown that chronic pain varies in intensity and most often is episodic $[4,5]$.

The etiology of NP is not fully understood, but some factors contributing to the development of persistent or recurrent NP have been identified. An initial trauma can trigger acute pain episodes transitioning into chronic NP [6]. Degenerative changes have also been suggested as an underlying cause $[7,8]$. However, tissue damage does not have to be present for chronic NP to develop [9]. Psychological factors, such as emotional trauma [10] or distress $[11,12]$, are also associated with the etiology of chronic NP. Signs of central sensitization and reduced inhibitory mechanisms [13] are often found among these patients, indicating increased sympathetic activation and reduced parasympathetic activation $[14,15]$. These two branches make up the Autonomic Nervous System (ANS) [16] and are functionally and anatomically distinct. They are responsible for maintaining homeostasis by regulating cells, tissues, and the function of organs. The ANS is regulated by supraspinal centers such as the limbic system, hypothalamus, and some brainstem nuclei, particularly the periaqueductal gray area [16].

Sympathetic and parasympathetic nervous system activity is altered in chronic pain conditions such as chronic low back and neck-shoulder pain, fibromyalgia, complex regional pain syndrome, and phantom limb pain $[17,18]$.

Disruption in autonomic balance can be measured using Heart Rate Variability (HRV) [17]. This is a marker of the vagal components of the heart's sinus node and measures the beat-to-beat changes in intervals $[19,20]$. In general, a high HRV indicates a well-functioning and adaptable ANS, while a low HRV indicates a poor-functioning ANS and is associated with a range of poor health outcomes [17, 21-23].

A range of treatment options exists for chronic NP [24]. One commonly used treatment method for musculoskeletal pain is Spinal Manipulative Therapy (SMT), defined as High-Velocity low Amplitude thrusts or mobilization of the spinal joints [25]. This technique is used by a range of professions [26] and has been shown to be effective for the treatment of NP, especially in combination with exercise therapy [27-29]. Based on a limited number of studies, the mechanisms of the pain-reducing effect of SMT proposed by Bialosky et al. [30] are thought to be multifactorial.

Several systematic reviews of the acute effects of SMT on ANS have been conducted [31-35]. However, these systematic reviews investigate a variety of SMT techniques and ANS outcome measures [34]. Only one systematic review includes assessment of risk of bias and evaluation of the quality of the outcome measures [34]. When summarizing these results, an acute (immediately after the intervention) ANS response can be observed with most SMT techniques. The evidence is of very low to moderate quality [31-35]. The exact mechanism of this immediate effect is not known.

There is a lack of well controlled Randomized Controlled Trials (RCT) investigating the long-term effects of SMT on HRV. The present study design was seen as the natural next step of the investigation into SMT and HRV. Two weeks was chosen and defined as longterm, based on previous research in the area investigating immediate effects. Because this study investigates patients potentially seeking care at a clinic, a pure placebo trial was not indicated [36]. Home stretching exercises were chosen as the comparison group because it was a viable treatment option for patients with neck pain. However, on the basis of previous research and current guidelines [24, 27, 37, 38], it was expected to have a smaller effect on pain and HRV than a combination of home stretching exercises and SMT. Stretching exercises are commonly used together with strengthening exercises and have been found to have a pain-reducing effect on persistent or recurrent NP, whilst home stretching exercises alone have been found to have a small or no beneficial effect [37]. In a study of women 
with chronic NP, the pain-reducing effect of stretching was similar to that of manual therapy alone [39].

The mechanism behind the pain-reducing effects of stretching is thought to be reduced neuronal discharge by inhibition of Golgi tendon organs [40]. Acute changes in the tension-length relationship in muscle tissue lead to greater flexibility, affected by the individual stretch tolerance [41-45] and possibly changes in the muscle's viscoelasticity [46]. Acute increases in HRV have also been seen with stretching exercises [47-51].

As recurrent or persistent NP is associated with imbalanced autonomic activity, e.g. reduced HRV, it is important to identify whether recommended treatments aimed at improving pain, such as SMT in combination with stretching exercises, also restore ANS balance in this patient group.

We hypothesized that four treatments with SMT and home stretching exercises are more effective in improving HRV than home stretching exercises alone.

This study aimed to investigate the effects of a 2-week treatment series consisting of (1) home stretching exercises and SMT versus (2) home stretching exercises alone on HRV in a population of patients with recurrent or persistent NP.

\section{Method}

The study is reported according to the CONSORT statement guidelines.

This was a multicenter study carried out in five multiprofessional clinics within the regional health service in Stockholm, Sweden. Clinics were chosen based on being similar in terms geographical location, having multi professional teams and subsidization of treatments. Chiropractors, dieticians, occupational therapists, and physiotherapists worked at all these clinics. All chiropractors were licensed by the Swedish National Board of Health and Welfare.

Recruitment began in January 2019, with the data collection ending in April 2020. The final follow-up questionnaires were answered in June 2020.

This article is the second publication reporting on the outcomes from an RCT, described in a published protocol [25] which provides detailed information about the study procedure and method.

\section{Recruitment}

Participants were recruited from patients seeking care at the participating clinics and collaborating GP clinics as well as advertisements in clinics' newsletters, Facebook, and local newspapers. The recruitment channels were adapted to fit the local procedures at each clinic. All the participants were screened for eligibility over the phone using a standardized check list and booked in for five treatments at the clinic by the primary researcher.

\section{Inclusion criteria}

- Presence of recurrent (at least one previous episode) and persistent (duration more than 6 months) NP [52].

- No chiropractic treatment in the previous 3 months.

- Minimum 18 years of age

- Able to read and write Swedish

\section{Exclusion criteria}

Conditions or medications that could affect the HRV measurements, such as

- diagnosed with cardiovascular disease

- diagnosed with hypertension

- diagnosed with diabetes type I or II

- pregnancy

- obesity (BMI > 30)

- on steroid medication

- on $\beta$-blocker medication

- on antidepressant medication

Participants were also excluded if they had

- serious, competing diagnoses such as cancer, infection, or recent severe trauma

- contra-indications to spinal manipulation, e.g. the recent development of headache or dizziness, previous drop-attacks, or acute cervical radiculopathy.

\section{Randomization}

SPSS version 20 (https://spss.software.informer.com/ $20.0 /$ ) was used to generate randomly permuted blocks of different sizes using a 1:1 allocation ratio by a research assistant. The same research assistant also prepared consecutively numbered sealed opaque envelopes containing participant information and group allocation. The sealed envelope was brought to the treating chiropractor at the first visit and opened there, providing the allocated treatment modality.

\section{Blinding}

The clinicians participating in the study were not blinded, as they were delivering the treatments. The researchers collecting the data were blinded to group allocation, and the main analysis was carried out by a statistician also blinded to group allocation. 
The participants did not know what treatment the other group was receiving. They were told that both groups were receiving treatments commonly used for persistent or recurrent NP. Thus, they were aware of the purpose of the trial but not the details of the "other" intervention. All participants (both treatment arms) received the same examination, support, and opportunity to ask questions about their condition. Treatment adherence was essential in both groups to ensure effective delivery of both interventions.

\section{Intervention}

All participants were booked for five visits to the clinic. The intervention included the four treatments and ended after the final measurements before the fifth visit. All visits had at least 2 days between them, with a maximum of two treatments each week for all participants.

One group received home stretching exercises (Additional file 5) and SMT (intervention group); the other received home stretching exercises only (control group). All clinicians provided treatments in both groups.

Intervention group On the basis of patient preferences and clinical impressions such as physical function and palpatory findings, the chiropractor tailored the type of SMT to the individual subject while conforming to the study's definition of SMT, i.e. High-Velocity low Amplitude thrusts or mobilization to the spinal joints [25]. Any area of the spine could be treated to allow for an individualized clinical approach by the clinicians, but also to allow for treatment when participants were reluctant to have treatment performed to the painful neck itself. Also, systematic reviews [31-35] have not been able to conclude on whether short-term sympathetic upregulation found with SMT is related to the spinal area being treated.

Control group The control group received the same examinations and verbal information as the intervention group for all visits, excluding any passive treatment. Thus, they discussed their exercises and pain with their chiropractor on subsequent visits.

All participants were asked to keep an exercise diary to monitor adherence to the home stretching exercises. Information from the patient files were obtained after the study period ended to control the adherence to SMT.

\section{Baseline}

During the first visit to the clinic (i.e. baseline), participants received written information about the study's purpose, data protection, contact information, and information about the follow-up questionnaires and daily SMS message. A consent form was signed before any measurements took place. They were then asked to fill in their first questionnaires on-site. This included demographics and questions concerning their recent caffeine and alcohol consumption, medication intake, and recent exercise.

After this, participants were placed in a quiet room with hearing protection. The first $5 \mathrm{~min}$ were then used as relaxation time to reduce any effect on HRV from external factors. During the next $5 \mathrm{~min}$, the HRV measurement was recorded in a resting state. The participants were then randomized and met their chiropractor for the initial consultation and first treatment session.

\section{Follow-up}

The participants were measured again prior to the third and fifth visits to the clinic (i.e. 1 and 2 weeks after the first treatment) for a total of 2 weeks. This was done to prevent any acute effect from treatment and ensure a standardized time interval between measurements. Three weekly follow up measurements of HRV were chosen to include sufficient amount of data without requiring too much time from the participants. At follow-up, the HRV measurement was recorded with the same procedure and conditions as at baseline.

\section{Outcome}

\section{Heart rate variability at rest}

HRV is recognized as a valid and reliable non-invasive measure of ANS and can be regarded as a biomarker for ANS regulation $[17,53]$. It has excellent reliability of indices reflecting central parasympathetic control over the heart rate either on a frequency- or time-domain analysis [54]. HRV at rest has been found to have moderate test-retest reliability in healthy adults [55]. HRV during standardized rest reflects resting autonomic cardiac modulation and is a valuable outcome in interventions targeting ANS in patients with pain [56]. Earlier research on HRV and persistent or recurrent NP have also used this strategy [52]. We measured HRV at rest for $5 \mathrm{~min}$. This is a standard short-term recording described by the Task Force Standards [56].

A heart rate monitor (Bodyguard2, Firstbeat Technologies Oy, Jyväskylä, Finland) was used to record R-R intervals at rest using a standard 2-lead ECG configuration (https://www.firstbeat.com/en/). This is a small portable device that is attached to the chest with Kendal Arbo H92SG electrodes. The device measures R-R intervals with a sampling rate of $1000 \mathrm{~Hz}$. The time series of R-R intervals were stored directly on the device and downloaded to a PC for off-line analysis of resting HRV. The Bodyguard 2 monitor has been found to produce results for indices of heart rate variability that are similar to the gold-standard laboratory electrocardiogram (Biopac MP150) during resting conditions [57]. 
The Taskforce of the European Society of Cardiology and the North American Society of Pacing and Electrophysiology [56] has developed standards of measurements to be used when investigating HRV. These were slightly adapted on the basis of previous research [58], the indices are summarized in Table 1.

\section{Data processing of HRV}

Prior to HRV analysis, all recordings of R-R intervals were visually inspected and manually cleaned for ectopic beats and artifacts by the primary researcher blinded to the treatment allocation, using Kubios software [59]. Both frequency domain and time parameters were analyzed using 5-min segments. Threshold-based beat correction algorithm testing with different sensitivity filters of R-R intervals was used. Several sensitivity filters exist in the software, ranging from 0.45 to $0.05 \mathrm{~s}$ differing from the local sample average [59]. Five-minute periods with more than $5 \%$ artifacts were excluded. This is in line with a previous study [60]. In this study, $6.7 \%$ of all analyzed data were excluded due to measurement error. Group difference on pain and disability in this study is reported in a separate article [61].

\section{Adverse reactions}

The participants were asked to report any adverse reactions from the first treatment by SMS message after their first visit, with an NRS-11 scale anchored by the descriptors 'No reaction' (0) and 'Worst reaction imaginable' (10) [62].

\section{Sample size}

Sample size was calculated a priori based on the outcome lnRMSSD, which is the primary time-domain measure, minimally affected by respiration [63]. The sample size was calculated based on values obtained from the article by Hallman et al. [52], and logarithmic values where used as we had reliable data on the means and distributions of the logarithmic values and wanted to base the study size on reliable information. A difference of $10-20 \%$ in $\operatorname{logRMSSD}$ has been considered clinically important [64]. Sixty participants were needed in each treatment arm to reach a power of $80 \%$ with a significant level of $5 \%$ to detect a change in lnRMSSD of $10 \%$ between groups [65].

\section{Ethics}

Stretching and SMT are considered to be safe and effective treatments for NP [66].

A unique number was assigned to each study subject by a research assistant at the time of inclusion. A code key linking the participant's unique number and ID is stored according to the Swedish National Board of Health and Welfare's requirements for the safekeeping of medical records.

\section{Statistical analysis}

Intention to treat analysis was used. Missing values were not imputed as only $11.8 \%$ of all observations were missing. These included dropouts, measurement error, and missed appointments, as reported in Fig. 1. A per protocol analysis was conducted as a sensitivity analysis to account for dropouts and group allocation mix ups.

The intervention effect on HRV indices (outcome) was analyzed using linear mixed-effects models with group, time, and the interaction as fixed effects, and a person specific random intercept. This interaction between group allocation and time can be interpreted as the difference in the groups' regression line for each time-point ( 1 and 2 weeks).

An additional analysis was performed, adjusting for age, and gender as stated in the protocol. The effect of baseline pain intensity on the changes in HRV is not

Table 1 Heart Rate Variability indices suggested by The Taskforce of the European Society of Cardiology and the North American Society of Pacing and Electrophysiology

\begin{tabular}{|c|c|c|c|}
\hline HRV indices & Indicator of & Domain measure & $\begin{array}{l}\text { Change that } \\
\text { improves } \\
\text { HRV }\end{array}$ \\
\hline R-R interval & Global HRV activity & Time & Increase \\
\hline $\begin{array}{l}\text { Root mean squared successive differences } \\
\text { between IBIs (RMSSD) }\end{array}$ & Parasympathetic (vagal) activity & Time & Increase \\
\hline The standard deviation of IBIs (SDNN) & Global HRV & Time & Increase \\
\hline Low frequency power (LF, 0.04-0.15 Hz) & $\begin{array}{l}\text { Baroreceptor-sympathetic and parasympathetic } \\
\text { cardiac activity }\end{array}$ & Frequency & Increase \\
\hline High frequency power $(\mathrm{HF}, 0.15-0.4 \mathrm{~Hz})$ & Parasympathetic (vagal) activity & Frequency & Increase \\
\hline LF/HF ratio & Sympathetic-to-parasympathetic balance & Frequency & Decrease \\
\hline Total power & Global HRV activity & Frequency & Increase \\
\hline
\end{tabular}

All indices are expected to increase with improved HRV except for the LF/HF ratio 


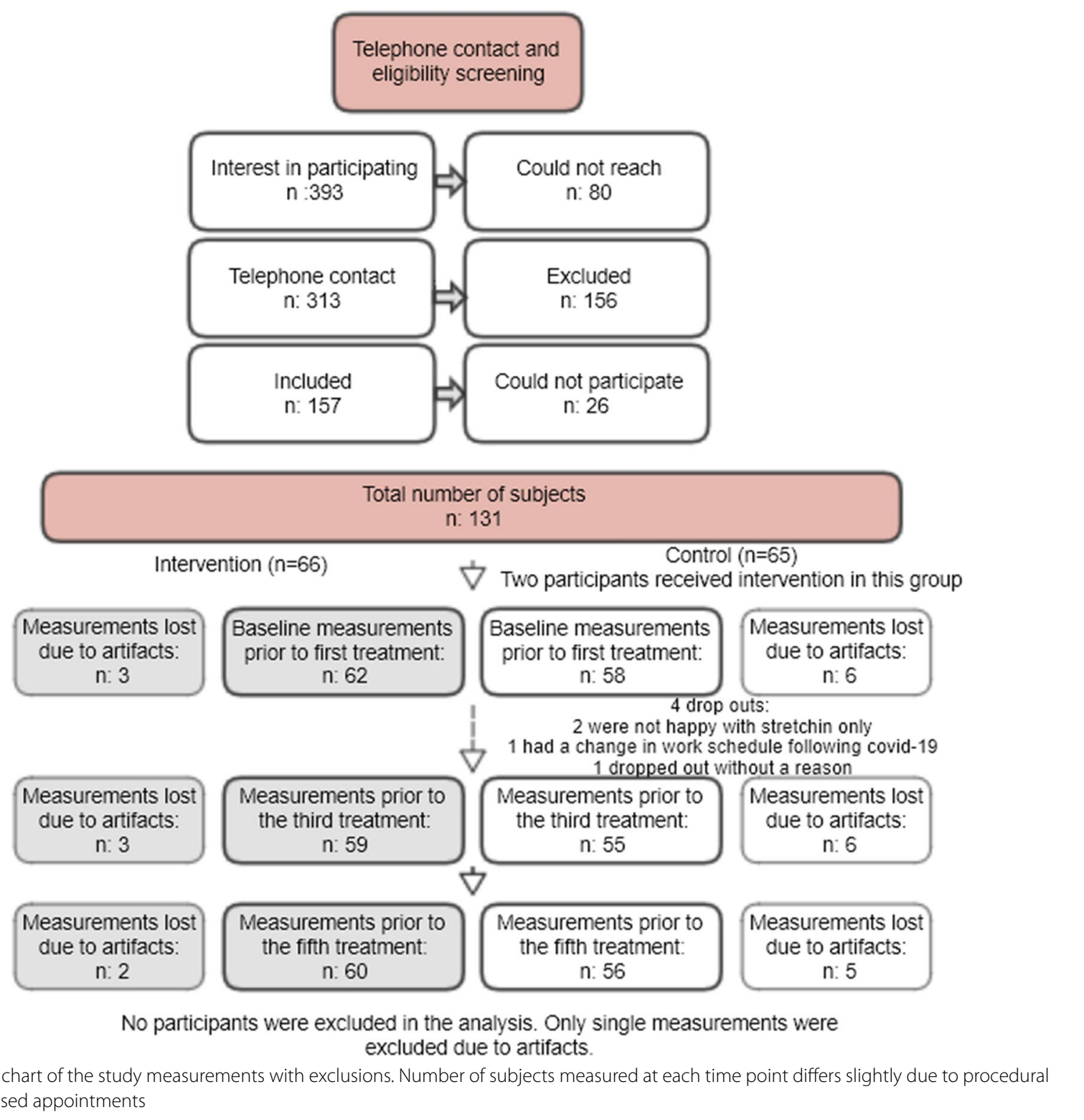

known, thus baseline differences in pain intensity were also adjusted for. All terms were entered at once.

Outliers were investigated with a sensitivity analysis, excluding all outliers visually disproportionally distant to the mean, but this analysis did not significantly affect the results.

A per-protocol analysis was also performed, which followed the same method as the primary analysis with repeated measures. This did not significantly affect the results.

The time effect for the total study population was calculated using a separate linear mixed-effects model without adding group allocation. This was done to investigate the change of the entire group because no group difference was observed. $P$ values smaller than 0.05 were considered significant. The analysis was performed using SPSS 27 [67] and Stata version 15 (StataCorp. 2017).

The graphical representations were done using a linear regression model representing the estimated difference between groups over 2 weeks.

\section{Results}

\section{Baseline}

A total of 131 patients were included in the study, 66 in the intervention group and 65 in the control group. After cleaning the HRV data, 25/350 measurements were lost 
due to insufficient ECG quality. See the flow chart (Fig. 1) for details.

The groups were similar at baseline, except for mean NP intensity (NRS-11), which was slightly higher for the intervention group, reported in Table 2 . This difference is not considered clinically relevant [68].

Some differences between the groups at baseline for the HRV outcome measures were also seen. HRV indices were slightly higher in the intervention group, reported in Table 3 together with mean differences.

Log and absolute values did not differ in precision or direction compared to the absolute values and yielded the same conclusions. The absolute values are presented in this article as they are easier to interpret and reported in similar research $[31,69,70]$. The results from the analysis of log values are found in Additional file 4 .

\section{Intervention effect on heart rate variability at rest}

The interaction effect between time and group is reported in Table 4, with a B-coefficient showing the difference in regression slope between the two groups for each time-point ( 1 and 2 weeks). The difference is visually available in Fig. 2. No statistically significant group effect was found for any of the HRV indices, with similar results in unadjusted and adjusted models. The adjusted model

Table 2 Demographics of the study population at baseline, $n=131$

\begin{tabular}{lll}
\hline & Intervention (66) & Control (65) \\
\hline Age, mean (sd) & $57(14.0)$ & $58(13.7)$ \\
Female, n (\%) & $37(56)$ & $36(55)$ \\
Baseline neck pain NRS-11, mean & 4.68 & 4.17 \\
Arm pain, n (\%) & $42(65)$ & $36(57)$ \\
Pain in the midback, n (\%) & $39(61)$ & $37(62)$ \\
Pain in the low back, n (\%) & $39(62)$ & $37(59)$ \\
Neck pain & & $1(2)$ \\
1. Less than 6 months, n (\%) & $0(0)$ & $10(16)$ \\
2. 6-12 months, n (\%) & $8(12)$ & $51(82)$ \\
3. Several years, n (\%) & $57(88)$ & \\
STarT back categories & & $48(79)$ \\
1. Low risk, n (\%) & $47(80)$ & $2(18)$ \\
2. Medium risk, n (\%) & $7(12)$ & $4(6)$ \\
3. High risk, n (\%) & $5(9)$ & $18(28)$ \\
Sick leave during previous year & & $41(63)$ \\
Does not work, n (\%) & $13(20)$ & \\
No, n (\%) & $47(71)$ & \\
Yes, between 1 and 7 days, n (\%) & $3(5)$ & $3(5)$ \\
Yes, between 8 and 14 days, n (\%) & $0(0)$ & \\
Yes, more than 15 days, n (\%) & & \\
\hline
\end{tabular}

Table 3 Means and mean differences of all indices of heart rate variability at rest $(n=123)$

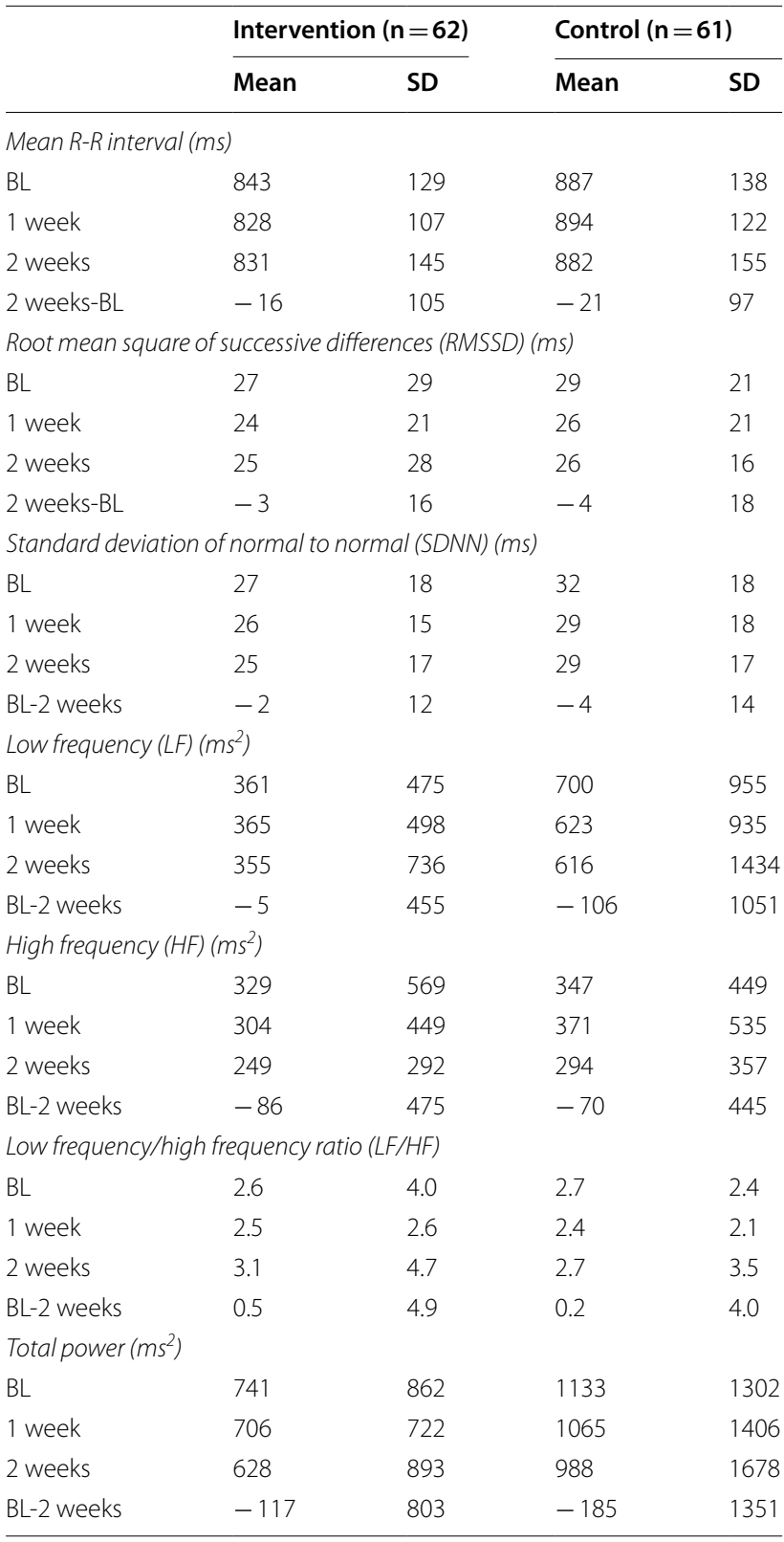

is found in Additional file 2. Additional details from the analysis are found in Additional files 1 and 2.

The time effect for the total study population is reported in Table 5, with the B-coefficient describing the regression slope for each time point ( 1 and 2 weeks). There was a slight decrease in all HRV indices over time except for LF/HF. However, only SDNN showed a statistically significant change $(\mathrm{B}=1.58, p=0.018)$, indicating reduced global HRV. Additional details from the analysis are found in Additional file 3. 
Table 4 Difference in the regression slope for each time point for intervention and control, control group as reference $(n=123)$

\begin{tabular}{lrcrr}
\hline Group $\times$ time & \multicolumn{4}{c}{ Treatment effect (unadjusted) } \\
\cline { 2 - 5 } & B & P value & $\mathbf{9 5 \% ~ C l} r$ & \\
\hline R-R $(\mathrm{ms})$ & 0.1 & 0.997 & -17.2 & 17.4 \\
RMSSD (ms) & 0.4 & 0.829 & -3.1 & 3.9 \\
SDNN (ms) & 0.8 & 0.548 & -1.8 & 3.4 \\
LF $\left(\mathrm{ms}^{2}\right)$ & 44.2 & 0.482 & -79.4 & 167.8 \\
HF $\left(\mathrm{ms}^{2}\right)$ & -12.5 & 0.746 & -88.3 & 63.3 \\
LF/HF & 0.2 & 0.498 & -0.5 & 0.9 \\
Total Power $\left(\mathrm{ms}^{2}\right)$ & 23.1 & 0.791 & -148.2 & 194.4 \\
\hline
\end{tabular}

$R-R$ mean $\mathrm{R}-\mathrm{R}$ interval, $R M S S D$ root mean square of successive differences, SDNN standard deviation of normal to normal, $L F$ low frequency, $H F$ high frequency, LF/HF LF/HF ratio

Table 5 Time effect for the total study sample, B indicating the regression line for each time point $(n=123)$

\begin{tabular}{|c|c|c|c|c|}
\hline \multirow[t]{2}{*}{$R-R$} & \multirow{2}{*}{$\begin{array}{l}\text { B } \\
-7.64\end{array}$} & \multirow{2}{*}{$\begin{array}{l}P \text { value } \\
0.082\end{array}$} & \multicolumn{2}{|l|}{$95 \% \mathrm{Cl}$} \\
\hline & & & -16.26 & 0.98 \\
\hline RMSSD & -1.48 & 0.098 & -3.23 & 0.28 \\
\hline SDNN & -1.58 & 0.018 & -2.88 & -0.28 \\
\hline LFms & -25.62 & 0.414 & -87.27 & 36.03 \\
\hline HFms & -36.75 & 0.056 & -74.51 & 1.02 \\
\hline LF/HF & 0.14 & 0.420 & -0.20 & 0.48 \\
\hline Total power & -71.94 & 0.089 & -157.28 & 13.40 \\
\hline
\end{tabular}

$R-R$ mean R-R interval, RMSSD root mean square of successive differences, SDNN standard deviation of normal to normal, $L F$ low frequency, $H F$ high frequency, LF/HF LF/HF ratio

A per protocol analysis did not change the overall estimates or precision and is therefore not reported here.

\section{Attrition}

Four participants dropped out during the first week. Two of these were not happy about receiving home stretching exercises only, one dropped out due to a change in their work schedule following Covid-19, and one canceled without giving a reason. All dropouts were in the control group. It was also found that two participants had received treatment even though they were part of the control group. They were subsequently moved to the intervention group in the per protocol analysis. One participant reported not to have had NP for longer than 6 months when answering the baseline questionnaire. The reason for this is not clear as all participants were screened prior to the initial clinic visit, which required a 6-month duration of NP. This was identified as a protocol deviation.

The study population showed good adherence to the home exercises. 118 out of 131 at baseline returned the training diary. Of these, $93.7 \%$ of the intervention group and $87.9 \%$ of the control group performed their home exercises at least 12 out of 14 days as seen in Table 6 .

All participants (100\%) in the intervention group received manual treatment as defined in this study.

\section{Adverse reactions}

Four intense adverse events (defined by $\geq 8 / 10$ (NRS-11) [71]) were reported in the study by three participants in the intervention group and one in the control group. There were no statistically significant differences in mean adverse reaction between the two groups $(p>0.05)$.

\section{Discussion}

We conducted a randomized clinical study of patients with persistent or recurrent NP to investigate the long-term effect on HRV of SMT and home stretching exercises versus home stretching exercises alone. Our findings indicated no significant effect on a wide range of HRV indices in both the time and frequency domains after 2 weeks of SMT and stretching compared to stretching alone. Nor was any overall improvement in HRV observed across groups. A per-protocol analysis did not significantly change the outcomes. This well-controlled RCT contributes to the literature by investigating the long-term effects of consecutive treatments of SMT and stretching. Previous studies have focused on acute effects during and directly following SMT.

The results may indicate that adding SMT to stretching does not improve HRV. This is at odds with previous research which has found mild to moderate evidence of the acute effect of SMT.

The study also measured changes in pain (NRS-11) between the groups, with no significant observed difference between the groups [61]. Pain (either acute

Table 6 Adherence to home stretching exercises as reported in exercise diaries

\begin{tabular}{|c|c|c|c|c|c|}
\hline $\begin{array}{l}\text { Number of days of having performed } \\
\text { stretching exercises (out of 14) }\end{array}$ & 10 & 11 & 12 & 13 & 14 \\
\hline Intervention, n (\%) & $4(6.3)$ & $0(0.0)$ & $10(15.9)$ & $10(15.9)$ & $39(61.9)$ \\
\hline Control, n (\%) & $0(0.0)$ & $7(12.7)$ & $6(10.9)$ & $6(10.9)$ & $36(65.5)$ \\
\hline
\end{tabular}



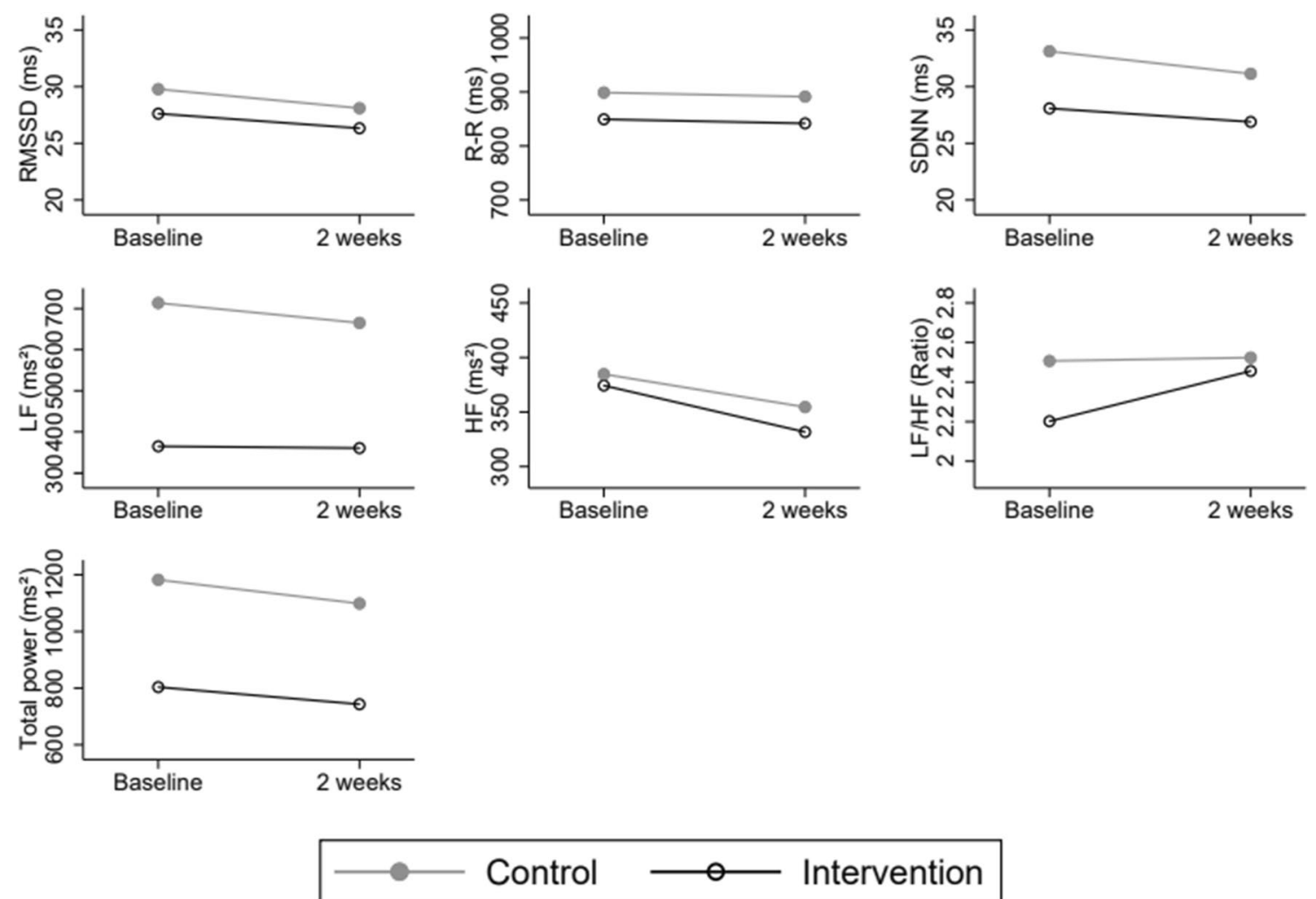

Fig. 2 HRV indices. Means for all heart rate variability indices at baseline, 1 week, and 2 weeks modelled using a linear regression model

or persistent) is known to be related to reduced HRV [17]. Thus, our results are in line with previous literature because neither pain nor HRV changed differently between the intervention and control groups [17].

Both groups demonstrated a slight worsening of HRV in the whole study sample, which could indicate a shift of the ANS towards sympathetic predominance. However, because the worsening of HRV was only significant for one out of seven HRV indices, it is possible that the interventions had no effect on HRV at all.

A possible explanation of the overall trend in reduced HRV might be related to the participants' expectations. As part of the test protocol, a conditioned pain modulation (CPM) test was included at the end of each visit. This is further explained in the study protocol [25]. The $\mathrm{CPM}$ test is an unpleasant procedure (hand submerged in cold water, $0-2{ }^{\circ} \mathrm{C}$ ). Even though we were careful about doing this after the HRV measurement, being aware of the painful nature of the CPM test that would come next could have influenced the HRV by increased sympathetic activity [72]. This would not be as evident on the first visit, when the participants had not yet experienced the unpleasant procedure, even though it had been explained to them.
Considerable variability in the individual changes in HRV was seen. This may suggest that responses varied considerably between patients. Whether this was due to individual differences in pain, physiological responses to treatments, or other factors remains unknown but warrants further investigation.

\section{Methodological considerations}

A 2-week intervention period might not have been sufficient to capture changes in HRV among people with persistent or recurrent NP. The length of the intervention period was based on three factors: (1) It is not considered ethical to keep the participants in a treatment group for too long if no improvement is seen [36]. (2) Previous studies have shown a significant effect of SMT on low back pain after 2 weeks [73]. (3) Previous studies have investigated the acute effects of SMT on HRV. Thus, a 2-week treatment period with a total of four treatments was considered long-term in relation to previous research.

The chiropractors were allowed to choose the appropriate SMT procedure for each subject, within the limits of the study, to allow a pragmatic approach. Many of the possible participants would be reluctant to participate if they had to receive a specific passive treatment, i.e. 
manual treatment to the painful neck itself. Patients' concerns and expectations are often taken into consideration in clinical practice and are one of the three cornerstones of evidence-based medicine [74]. The choice of treatment technique was recorded and will be presented in a separate publication.

\section{Weaknesses}

Because a large part of the study sample was recruited through clinics the participants themselves had chosen, perhaps based on previous contact with the clinic, selection bias may have skewed the results. This was addressed in the study design when clinics were chosen based on, among other things, having multi professional teams. Thus, participants recruited at the clinic would be equally likely to have been in contact with other health care providers at the clinic. This sort of bias is, however, difficult to completely remove from a trial. Selection bias can be assumed to be considerable if the participants had seen the study therapist before. Our data do not demonstrate that this was the case.

It was impossible to blind the chiropractors performing the interventions. This is a possible source of bias because the therapists might favor the intervention group. However, the study was designed to control this by providing written and oral information to clinicians about how to interact with the study participants. Our data do not demonstrate that such bias influenced the results.

HRV typically fluctuates during the day [75]. Due to the study's pragmatic design, it was difficult to book the participants at the same time of the day for each measurement. All participants were booked in during the clinic's opening hours (0700-1600).

The ANS is influenced by internal and external factors such as grief, relationship issues, and other unknown underlying diseases that cannot be controlled for. Also, measurement errors and moderate reliability of HRV could possibly have affected the results. The randomized design should balance such factors between groups. However, it was seen that the intervention group had generally higher HRV than the control group at baseline.

It is possible that persistent or recurrent NP is more resistant to SMT than low back pain. Hence, four treatments over 2 weeks might not have been sufficient to see the same improvements for this patient group as observed for low back pain patients [73]. Long-term follow ups might be needed.

As the sample size was based on a calculation of between group differences, repeated measures might have affected the power of this study, but the effect is unknown.

\section{Strengths}

The main strength of this article is the randomized controlled design.

The study participants did not know what intervention the other group was receiving, and the research assistant and statistician were blinded to group allocation.

Both groups received the same number of treatments and amount of attention from the chiropractors. The control group went through a palpatory examination at each visit, even though treatment was not intended. This was important to balance the contextual effects. The study was pragmatic by nature and mirrored typical treatment strategies.

HRV is recognized as a valid and reliable non-invasive measure of ANS. However, about $40 \%$ of a single HRV measurement variance can be explained by the situational effects and person-situation interaction [53]. To address this, a protocol of the procedure was produced and implemented in all participating clinics, before commencing the data collection. The specific measurements and test procedures were also practiced by the two researchers performing them and individually planned for each clinic. The two researchers observed each other to calibrate the measurements and instructions given to patients. This consistency in HRV measurements is considered a major strength of this study. Finally, similar conditions were maintained for all measurements. These include temperature in the room, elimination of disturbing noises, and no alcohol, heavy exercise, or caffeine before the measurements. All these measures were taken to minimize situational effects and person-situation interaction. The number of dropouts was small and adherence to treatment was excellent.

The result from this study indicates that the observed immediate effects of SMT on HRV have no clinical implications over 2 weeks for this patient group.

Future research might examine the relationship between changes in pain and HRV during treatment, include patients with higher pain intensity, or provide a longer treatment period.

\section{Conclusion}

Adding SMT to a 2-week stretching protocol did not result in improved HRV in this well-controlled RCT. Previous findings about the immediate effects on HRV of SMT do not seem to be transferable to a long-term effect, 
based on the current trial using a longer follow-up time period.

\author{
Abbreviations \\ NP: Neck pain; SMT: Spinal Manipulative Therapy; HRV: Heart Rate Variability; \\ NRS-11: 11-Point numeric rating scale; ANS: Autonomic nervous system.
}

\section{Supplementary Information}

The online version contains supplementary material available at https://doi. org/10.1186/s12998-021-00406-0.

Additional file 1. Difference in the regression slope for each time point for intervention and control, control group as reference with all details from the regression model $(n=123)$ (Unadjusted).

Additional file 2. Difference in the regression slope for each time point for intervention and control, control group as reference with all details from the regression model $(n=123)$ (Adjusted for age, sex, and NRS baseline values).

Additional file 3. Time effect for the total study sample, B indicating the regression line for each time point with all details from the regression model $(n=123)$

Additional file 4. Unadjusted file 4. Difference in the regression slope for each time point for intervention and control, control group as reference ( $n$ $=123)$, unadjusted In values.

Additional file 5. Home stretching exercises.

\section{Acknowledgements}

Thanks to www.kiropraktorcentrum.com for the permission to use their photos in the exercise diary. A special thanks to Anna Warnqvist for assisting in performing the statistical analysis, and in preparing figures.

\section{Authors' contributions}

AGB designed the study, wrote the main manuscript, performed the main statistical analysis, and prepared tables and text. IA designed the study and assisted in the interpretation of results and the writing of the manuscript. AE assisted in designing the study, interpretation of results, and writing of the manuscript. DH assisted in designing the study, interpreting results, and reviewed the manuscript. All authors read and approved the final manuscript.

\section{Funding}

Open access funding provided by Karolinska Institute. IKON — (Institute for Chiropractic and Neuromusculoskeletal Research) provided financial support to this article. The funding body had no role in the design of the study, analysis or interpretation of data, or writing of the manuscript.

\section{Availability of data and materials}

The data that support the findings of this study are available from Karolinska Institutet. Restrictions apply to the availability of these data, which were used under license for the current study, and so are not publicly available. With the permission of Karolinska Institutet, data are available from the authors upon reasonable request.

\section{Declarations}

Ethical approval and consent to participate

Signed informed consent was obtained from all participants. The Regional Ethical Review Board (Stockholm) [76] approved this study (reference approval no. 2018/2137-31). All methods were performed in accordance with relevant guidelines and regulations.

\section{Consent for publication}

Written consent for the use of the photos of home stretching exercises was obtained.

\section{Competing interests}

Iben Axén is Co-Editor in Chief in Chiropractic and Manual Therapies. She has no influence over the review process of this article.

\section{Author details}

'Department of Environmental Medicine, Division of Intervention and Implementation Research for Worker Health, Karolinska Institutet, Nobels väg 13, 17177 Stockholm, Sweden. ${ }^{2}$ Centre for Musculoskeletal Research (CBF), Department of Occupational Health Sciences and Psychology, University of Gävle, Gävle, Sweden.

Received: 4 October 2021 Accepted: 18 November 2021

Published online: 29 November 2021

\section{References}

1. Treede R-D, RiefW, Barke A, Aziz Q, Bennett MI, Benoliel R, et al. A classification of chronic pain for ICD-11. Pain. 2015;156:1003-7. https://doi.org/ 10.1097/j.pain.0000000000000160.

2. Hurwitz EL, Randhawa K, Yu H, Cote P, Haldeman S. The Global Spine Care Initiative: a summary of the global burden of low back and neck pain studies. Eur Spine J Off Publ Eur Spine Soc Eur Spinal Deform Soc Eur Sect Cerv Spine Res Soc. 2018;27:796-801. https://doi.org/10.1007/ s00586-017-5432-9.

3. Safiri S, Kolahi AA, Hoy D, Buchbinder R, Mansournia MA, Bettampadi D et al. Global, regional, and national burden of neck pain in the general population, 1990-2017: systematic analysis of the Global Burden of Disease Study 2017. BMJ (Clin Res Ed). 2020;368: m791. https://doi.org/ 10.1136/bmj.m791.

4. Axén I, Leboeuf-Yde C. Trajectories of low back pain. Best Pract Res Clin Rheumatol. 2013;27:601-12. https://doi.org/10.1016/j.berh.2013.10. 004.

5. Raffaeli WTM, Corraro A, Malafoglia V, Ilari S, Balzani E, Bonci A. Chronic pain: what does it mean? A review on the use of the term chronic pain in clinical practice. J Pain Res. 2021. https://doi.org/10.2147/JPR.S3031 86

6. Nederhand MJ, Hermens HJ, IJzerman MJ, Turk DC, Zilvold G. Chronic neck pain disability due to an acute whiplash injury. Pain. 2003;102:63-71. https://doi.org/10.1016/s0304-3959(02)00340-8.

7. Evans G. Identifying and treating the causes of neck pain. Med Clin. 2014;98:645-61. https://doi.org/10.1016/j.mcna.2014.01.015.

8. Peng B, DePalma MJ. Cervical disc degeneration and neck pain. J Pain Res 2018;11:2853-7. https://doi.org/10.2147/jpr.S180018.

9. van Wilgen $C P$, Keizer D. The sensitization model to explain how chronic pain exists without tissue damage. Pain Manag Nurs Off J Am Soc Pain Manag Nurses. 2012;13:60-5. https://doi.org/10.1016/j.pmn.2010.03.001.

10. Ravn SL, Vaegter HB, Cardel T, Andersen TE. The role of posttraumatic stress symptoms on chronic pain outcomes in chronic pain patients referred to rehabilitation. J Pain Res. 2018;11:527-36. https://doi.org/10. 2147/jpr.S155241.

11. Buscemi V, Chang WJ, Liston MB, McAuley JH, Schabrun S. The role of psychosocial stress in the development of chronic musculoskeletal pain disorders: protocol for a systematic review and meta-analysis. Syst Rev. 2017;6:224. https://doi.org/10.1186/s13643-017-0618-0.

12. Gerdle B, Åkerblom S, Stålnacke B-M, Jansen G, Enthoven P, Ernberg M, et al. The importance of emotional distress, cognitive behavioural factors and pain for life impact at baseline and for outcomes after rehabilitation-a SQRP study of more than 20,000 chronic pain patients. Scand J Pain. 2019. https://doi.org/10.1515/sjpain-2019-0016.

13. Nijs J, Van Houdenhove B, Oostendorp RA. Recognition of central sensitization in patients with musculoskeletal pain: application of pain neurophysiology in manual therapy practice. Man Ther. 2010;15:135-41. https://doi.org/10.1016/j.math.2009.12.001.

14. Gockel $M$, Lindholm $H$, Alaranta $H$, Viljanen $A$, Lindquist $A$, Lindholm $T$. Cardiovascular functional disorder and stress among patients having neck-shoulder symptoms. Ann Rheum Dis. 1995;54:494-7. https://doi. org/10.1136/ard.54.6.494.

15. Hallman DM, Ekman AH, Lyskov E. Changes in physical activity and heart rate variability in chronic neck-shoulder pain: monitoring during work 
and leisure time. Int Arch Occup Environ Health. 2014;87:735-44. https:// doi.org/10.1007/s00420-013-0917-2.

16. Jänig W. The integrative action of the autonomic nervous system; 2006.

17. Tracy LM, loannou L, Baker KS, Gibson SJ, Georgiou-Karistianis N, Giummarra MJ. Meta-analytic evidence for decreased heart rate variability in chronic pain implicating parasympathetic nervous system dysregulation. Pain. 2016;157:7-29. https://doi.org/10.1097/j.pain.0000000000000360.

18. Bandeira PM, Reis FJJ, Sequeira VCC, Chaves ACS, Fernandes O, ArrudaSanchez T. Heart rate variability in patients with low back pain: a systematic review. Scand J Pain. 2021. https://doi.org/10.1515/sjpain-2021-0006.

19. Heart rate variability. Standards of measurement, physiological interpretation, and clinical use. Task Force of the European Society of Cardiology and the North American Society of Pacing and Electrophysiology. Eur Heart J. 1996;17:354-81

20. Berntson GG, Bigger JT Jr, Eckberg DL, Grossman P, Kaufmann PG, Malik $M$, et al. Heart rate variability: origins, methods, and interpretive caveats. Psychophysiology. 1997;34:623-48. https://doi.org/10.1111/j.1469-8986 1997.tb02140.x

21. Appelhans $B M$, Luecken $L$. Heart rate variability as an index of regulated emotional responding. Rev Gen Psychol. 2006;10:229-40. https://doi.org/ 10.1037/1089-2680.10.3.229.

22. Evrengül $H$, Dursunoglu D, Cobankara V, Polat B, Seleci D, Kabukçu S, et al. Heart rate variability in patients with rheumatoid arthritis. Rheumatol Int. 2004;24:198-202. https://doi.org/10.1007/s00296-003-0357-5.

23. Maser RE, Lenhard MJ. An overview of the effect of weight loss on cardiovascular autonomic function. Curr Diabetes Rev. 2007;3:204-11. https:// doi.org/10.2174/157339907781368931.

24. Hurwitz EL, Carragee EJ, van der Velde G, Carroll LJ, Nordin M, Guzman $J$, et al. Treatment of neck pain: noninvasive interventions: results of the bone and joint decade 2000-2010 task force on neck pain and its associated disorders. Spine (Phila Pa 1976). 2008;33:S123-52. https://doi.org/10. 1097/BRS.0b013e3181644b1d.

25. Galaasen Bakken A, Eklund A, Hallman DM, Axén I. The effect of spinal manipulative therapy and home stretching exercises on heart rate variability in patients with persistent or recurrent neck pain: a randomized controlled trial. Trials. 2019;20:590. https://doi.org/10.1186/ s13063-019-3678-8.

26. Pettman E. A history of manipulative therapy. J Man Manip Ther. 2007;15:165-74. https://doi.org/10.1179/106698107790819873.

27. Maiers M, Bronfort G, Evans R, Hartvigsen J, Svendsen K, Bracha Y, et al. Spinal manipulative therapy and exercise for seniors with chronic neck pain. Spine J Off J N Am Spine Soc. 2014;14:1879-89. https://doi.org/10. 1016/j.spinee.2013.10.035.

28. Coulter ID, Crawford C, Vernon H, Hurwitz EL, Khorsan R, Booth MS, et al. Manipulation and mobilization for treating chronic nonspecific neck pain: a systematic review and meta-analysis for an appropriateness panel. Pain Phys. 2019;22:E55-e70.

29. Miller J, Gross A, D'Sylva J, Burnie SJ, Goldsmith CH, Graham N, et al. Manual therapy and exercise for neck pain: a systematic review. Man Ther. 2010;15:334-54. https://doi.org/10.1016/j.math.2010.02.007.

30. Bialosky JE, Bishop MD, Price DD, Robinson ME, George SZ. The mechanisms of manual therapy in the treatment of musculoskeletal pain: a comprehensive model. Man Ther. 2009;14:531-8. https://doi.org/10. 1016/j.math.2008.09.001.

31. Amoroso Borges BL, Bortolazzo GL, Neto HP. Effects of spinal manipulation and myofascial techniques on heart rate variability: a systematic review. J Bodyw Mov Ther. 2018;22:203-8. https://doi.org/10.1016/j.jbmt. 2017.09.025.

32. Kingston L, Claydon L, Tumilty S. The effects of spinal mobilizations on the sympathetic nervous system: a systematic review. Man Ther. 2014;19:281-7. https://doi.org/10.1016/j.math.2014.04.004.

33. Chu J, Allen DD, Pawlowsky S, Smoot B. Peripheral response to cervical or thoracic spinal manual therapy: an evidence-based review with meta analysis. J Man Manip Ther. 2014;22:220-9. https://doi.org/10.1179/20426 18613 Y.0000000062.

34. Picchiottino M, Leboeuf-Yde C, Gagey O, Hallman DM. The acute effects of joint manipulative techniques on markers of autonomic nervous system activity: a systematic review and meta-analysis of randomized sham-controlled trials. Chiropr Man Ther. 2019;27:17. https://doi.org/10. 1186/s12998-019-0235-1.
35. Wirth B, Gassner A, de Bruin ED, Axen I, Swanenburg J, Humphreys BK, et al. Neurophysiological effects of high velocity and low amplitude spinal manipulation in symptomatic and asymptomatic humans: a systematic literature review. Spine (Phila Pa 1976). 2019;44:E914-e26. https:// doi.org/10.1097/brs.0000000000003013.

36. Saunders J, Wainwright P. Risk, Helsinki 2000 and the use of placebo in medical research. Clin Med (Lond). 2003;3:435-9. https://doi.org/10.7861/ clinmedicine.3-5-435.

37. Gross AR, Paquin JP, Dupont G, Blanchette S, Lalonde P, Cristie T, et al. Exercises for mechanical neck disorders: a Cochrane review update. Man Ther. 2016;24:25-45. https://doi.org/10.1016/j.math.2016.04.005.

38. Parikh P, Santaguida P, Macdermid J, Gross A, Eshtiaghi A. Comparison of CPG's for the diagnosis, prognosis and management of non-specific neck pain: a systematic review. BMC Musculoskelet Disord. 2019;20:81. https:// doi.org/10.1186/s12891-019-2441-3.

39. Ylinen J, Kautiainen $\mathrm{H}$, Wiren $\mathrm{K}$, Hakkinen A. Stretching exercises vs manual therapy in treatment of chronic neck pain: a randomized, controlled cross-over trial. J Rehabil Med. 2007;39:126-32. https://doi.org/10. 2340/16501977-0015.

40. Phadke A, Bedekar N, Shyam A, Sancheti P. Effect of muscle energy technique and static stretching on pain and functional disability in patients with mechanical neck pain: a randomized controlled trial. Hong Kong Physiother J. 2016;35:5-11. https://doi.org/10.1016/j.hkpj.2015.12.002.

41. Page P. Current concepts in muscle stretching for exercise and rehabilitation. Int J Sports Phys Ther. 2012;7:109-19.

42. Ylinen J, Kankainen T, Kautiainen $\mathrm{H}$, Rezasoltani A, Kuukkanen T, Hakkinen A. Effect of stretching on hamstring muscle compliance. J Rehabil Med. 2009;41:80-4. https://doi.org/10.2340/16501977-0283.

43. Halbertsma JP, Goeken LN. Stretching exercises: effect on passive extensibility and stiffness in short hamstrings of healthy subjects. Arch Phys Med Rehabil. 1994;75:976-81.

44. Ben M, Harvey LA. Regular stretch does not increase muscle extensibility: a randomized controlled trial. Scand J Med Sci Sports. 2010;20:136-44. https://doi.org/10.1111/j.1600-0838.2009.00926.x.

45. Law RY, Harvey LA, Nicholas MK, Tonkin L, De Sousa M, Finniss DG. Stretch exercises increase tolerance to stretch in patients with chronic musculoskeletal pain: a randomized controlled trial. Phys Ther. 2009;89:1016-26. https://doi.org/10.2522/pti.20090056.

46. Guissard N, Duchateau J, Hainaut K. Mechanisms of decreased motoneurone excitation during passive muscle stretching. Exp Brain Res. 2001;137:163-9. https://doi.org/10.1007/s002210000648.

47. Inami T, Shimizu T, Baba R, Nakagaki A. Acute changes in autonomic nerve activity during passive static stretching. Am J Sports Sci Med. 2014:2:166-70. https://doi.org/10.12691/ajssm-2-4-9.

48. Wong A, Figueroa A. Effects of acute stretching exercise and training on heart rate variability: a review. J Strength Cond Res. 2021;35:1459-66. https://doi.org/10.1519/jsc.00000000000003084.

49. Farinatti PT, Brandão C, Soares PP, Duarte AF. Acute effects of stretching exercise on the heart rate variability in subjects with low flexibility levels. J Strength Cond Res. 2011;25:1579-85. https://doi.org/10.1519/JSC.0b013 e3181e06ce1.

50. Mueck-Weymann M, Janshoff G, Mueck H. Stretching increases heart rate variability in healthy athletes complaining about limited muscular flexibility. Clin Auton Res. 2004;14:15-8. https://doi.org/10.1007/ s10286-004-0123-0.

51. Saito T, Hono T, Miyachi M. Effects of stretching on cerebrocortical and autonomic nervous system activities and systemic circulation. J Phys Med. 2001:12:2-9.

52. Hallman DM, Olsson EMG, von Schéele B, Melin L, Lyskov E. Effects of heart rate variability biofeedback in subjects with stress-related chronic neck pain: a pilot study. Appl Psychophysiol Biofeedback. 2011;36:71-80. https://doi.org/10.1007/s10484-011-9147-0.

53. Bertsch K, Hagemann D, Naumann E, Schachinger H, Schulz A. Stability of heart rate variability indices reflecting parasympathetic activity. Psychophysiology. 2012;49:672-82. https://doi.org/10.1111/j.1469-8986.2011. 01341.x.

54. Skotte JH, Kristiansen J. Heart rate variability analysis using robust period detection. Biomed Eng Online. 2014;13:138. https://doi.org/10.1186/ 1475-925X-13-138 
55. Sandercock GR, Bromley PD, Brodie DA. The reliability of short-term measurements of heart rate variability. Int J Cardiol. 2005;103:238-47. https:// doi.org/10.1016/j.jijcard.2004.09.013.

56. Heart rate variability: standards of measurement, physiological interpretation and clinical use. Task Force of the European Society of Cardiology and the North American Society of Pacing and Electrophysiology. Circulation. 1996;93:1043-65.

57. Palmer AR, Distefano R, Leneman K, Berry D. Reliability of the BodyGuard2 (FirstBeat) in the detection of heart rate variability. Appl Psychophysiol Biofeedback. 2021;46:251-8. https://doi.org/10.1007/ s10484-021-09510-6.

58. Hallman DM, Holtermann A, Søgaard K, Krustrup P, Kristiansen J, Korshøj M. Effect of an aerobic exercise intervention on cardiac autonomic regulation: a worksite RCT among cleaners. Physiol Behav. 2017;169:90-7. https://doi.org/10.1016/j.physbeh.2016.11.031.

59. Kubios [cited 2021]. https://www.kubios.com/.

60. Hallman DM, Sato T, Kristiansen J, Gupta N, Skotte J, Holtermann A. Prolonged sitting is associated with attenuated heart rate variability during sleep in blue-collar workers. Int J Environ Res Public Health. 2015;12:14811-27. https://doi.org/10.3390/ijerph121114811.

61. Galaasen Bakken A, Eklund A, Hallman DM, Warnqvist A, O'Neill S, Axén I. The effect of Spinal Manipulative therapy and home stretching exercises on pain and disability in patients with persistent or recurrent neck pain; a randomized controlled trial. Submitted to BMC musculoskeletal disorders 10042021. 2021.

62. SMS-track [cited 202115 of October]. https://www.sms-track.com

63. Shaffer F, Ginsberg JP. An overview of heart rate variability metrics and norms. Front Public Health. 2017. https://doi.org/10.3389/fpubh.2017. 00258.

64. Hallman DM, Srinivasan D, Mathiassen SE. Short- and long-term reliability of heart rate variability indices during repetitive low-force work. Eur J Appl Physiol. 2015;115:803-12. https://doi.org/10.1007/ s00421-014-3066-8.

65. de Araujo FX, Scholl Schell M, Ferreira GE, Pessoa MDV, de Oliveira LR, Borges $B G$, et al. Autonomic function and pressure pain threshold following thoracic mobilization in asymptomatic subjects: a randomized controlled trial. J Bodyw Mov Ther. 2018;22:313-20. https://doi.org/10. 1016/j.jbmt.2017.09.005

66. Carnes D, Mars TS, Mullinger B, Froud R, Underwood M. Adverse events and manual therapy: a systematic review. Man Ther. 2010;15:355-63. https://doi.org/10.1016/j.math.2009.12.006.

67. SPSS [cited 2021]. https://www.ibm.com/products/spss-statistics.

68. Farrar JT, Young JP Jr, LaMoreaux L, Werth JL, Poole RM. Clinical importance of changes in chronic pain intensity measured on an 11-point numerical pain rating scale. Pain. 2001;94:149-58. https://doi.org/10. 1016/s0304-3959(01)00349-9.

69. de Araujo FX, Scholl Schell M, Ferreira GE, Pessoa MDV, de Oliveira LR, Borges BG, et al. Autonomic function and pressure pain threshold following thoracic mobilization in asymptomatic subjects: a randomized controlled trial. J Bodyw Mov Ther. 2017. https://doi.org/10.1016/j.jbmt. 2017.09.005.

70. Younes M, Nowakowski K, Didier-Laurent B, Gombert M, Cottin F. Effect of spinal manipulative treatment on cardiovascular autonomic control in patients with acute low back pain. Chiropr Man Ther. 2017;25:33. https:// doi.org/10.1186/s12998-017-0167-6.

71. Rubinstein SM, Leboeuf-Yde C, Knol DL, de Koekkoek TE, Pfeifle CE, van Tulder MW. The benefits outweigh the risks for patients undergoing chiropractic care for neck pain: a prospective, multicenter, cohort study. J Manip Physiol Ther. 2007;30:408-18. https://doi.org/10.1016/j.jmpt.2007. 04.013.

72. Hallman DM, Lindberg LG, Arnetz BB, Lyskov E. Effects of static contraction and cold stimulation on cardiovascular autonomic indices, trapezius blood flow and muscle activity in chronic neck-shoulder pain. Eur J Appl Physiol. 2011;111:1725-35. https://doi.org/10.1007/s00421-010-1813-z.

73. Leboeuf-Yde C, Axén I, Jones JJ, Rosenbaum A, Løvgren PW, Halasz L, et al. The Nordic back pain subpopulation program: the long-term outcome pattern in patients with low back pain treated by chiropractors in Sweden. J Manip Physiol Ther. 2005;28:472-8. https://doi.org/10.1016/j.jmpt. 2005.07.003.

74. Straus S, Glasziou PW, Richardson SR, Haynes B. Evidence-Based Medicine, 5th Edition, How to Practice and Teach EBM: Elsevier; 2018. 336 p
75. Jarczok MN, Guendel H, McGrath JJ, Balint EM. Circadian rhythms of the autonomic nervous system: scientific implication and practical implementation, chronobiology. The Science of Biological Time Structure, Pavol Svorc, IntechOpen. 2019. Doi: https://doi.org/10.5772/intechopen.86822.

76. https://etikprovningsmyndigheten.se/. Available from: https://etikprovni ngsmyndigheten.se/.

\section{Publisher's Note}

Springer Nature remains neutral with regard to jurisdictional claims in published maps and institutional affiliations.
Ready to submit your research? Choose BMC and benefit from:

- fast, convenient online submission

- thorough peer review by experienced researchers in your field

- rapid publication on acceptance

- support for research data, including large and complex data types

- gold Open Access which fosters wider collaboration and increased citations

- maximum visibility for your research: over 100M website views per year

At BMC, research is always in progress.

Learn more biomedcentral.com/submissions 